\title{
EOmmun|HAlin, Communication et organisation
}

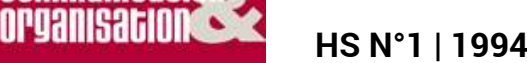

Communication et hôpital - Analyse et diagnostic

\section{Les enjeux de la communication à l'hôpital}

Jean-François Tome

\section{OpenEdition}

Journals

Édition électronique

URL : http://journals.openedition.org/communicationorganisation/2984

DOI : 10.4000/communicationorganisation.2984

ISSN : 1775-3546

Éditeur

Presses universitaires de Bordeaux

Édition imprimée

Date de publication : 1 mai 1994

ISSN : 1168-5549

\section{Référence électronique}

Jean-François Tome, « Les enjeux de la communication à l'hôpital », Communication et organisation [En ligne], HS N¹ | 1994, mis en ligne le 27 mars 2012, consulté le 01 mai 2019. URL : http://

journals.openedition.org/communicationorganisation/2984 ; DOI : 10.4000/

communicationorganisation.2984

Ce document a été généré automatiquement le 1 mai 2019.

(c) Presses universitaires de Bordeaux 


\title{
Les enjeux de la communication à l'hôpital
}

\author{
Jean-François Tome
}

1 Il n'est pas anodin que la création de services de communication dans les hôpitaux dans les années 80 corresponde à la mise en place du budget global : c'est-à-dire au moment où l'on commençait à connaître à l'hôpital les contraintes budgétaires alors que le besoin de santé était sans cesse croissant. Les problèmes économiques ont donc touché de plein fouet l'hôpital: comment faire en sorte que le soin à l'hôpital ne soit pas un soin à plusieurs vitesses?

2 Dans ce contexte, la communication est devenue le moyen diront certains, l'alibi diront les autres, d'un accompagnement de ces transformations économiques. Un accompagnement des choix que l'hôpital doit faire s'il ne veut pas transformer la santé en parent pauvre du service public.

3 Il est clair que la communication à l'hôpital ne peut pas ne pas tenir compte de ces contraintes qui impliquent une réorganisation des activités et des pratiques de soins, mais surtout une modification des comportements des gens.

4 L'hôpital est en effet un monde un peu particulier en raison de ses diversités culturelles. Celles-ci ressortent de la coexistence de métiers différents, et probablement de logiques de pouvoirs diverses.

5 L'enjeu majeur de la communication sera de travailler à la reconnaissance: reconnaissance des métiers, reconnaissance des gens eux-mêmes. Or, la reconnaissance n'a d'intérêt que si elle est mutuelle. C'est un travail de tous les instants, où l'on ne doit pas économiser sa peine et son temps.

6 La communication à l'hôpital s'analyse donc au travers d'un enjeu économique et d'un enjeu culturel. 


\section{Communication et enjeu économique : La recherche de la cohérence}

7 Aujourd'hui, nos budgets hospitaliers suivent à peine l'évolution de l'inflation; les contraintes qui pèsent sur l'hôpital font que, dans la réalité, ces budgets sont en diminution constante selon la logique «faire autant et plus avec un budget qui évolue très lentement voire stagne ».

8 Je ne ferai pas du misérabilisme hospitalier, mais il est essentiel de prendre en compte cet élément pour animer une communication au sein de l'hôpital. On peut ainsi se demander quelle va être la ressource que les responsables hospitaliers, les médecins et les autres vont trouver dans la communication pour gérer cet enjeu économique?

9 Un des premiers moyens est une certaine recherche de la cohérence : cohérence des choix économiques, cohérence des choix stratégiques mais aussi cohérence des choix individuels au sein de l'hôpital.

10 Il y a quelques années je rencontrai un chef de service hospitalier qui me disait: "L'hôpital ce n'est pas un orchestre mais un ensemble de solistes". Organiser la communication au sein de l'hôpital c'est probablement s'efforcer de rendre cohérent cet univers de solistes.

11 La loi hospitalière de 1991 fonde toute politique hospitalière sur la notion de projet, sur une véritable démarche stratégique. Celle-ci est le point de départ de toute communication efficace sans cesse à la recherche d'un système d'intégration suffisamment clair.

12 Tout le travail de communication de l'hôpital va permettre le passage d'une stratégie fondée sur une multiplicité de micro-décisions (concept de "stratégie émergente ») à une stratégie fondée sur un jeu triangulaire.

13 Pour être plus clair revenons à notre univers de solistes. Pendant des années la stratégie hospitalière jusqu'à la fin des années 70 a vécu pratiquement sans contrainte économique et l'on pouvait «laisser faire»: laisser faire le cardiologue qui voulait évoluer vers la meilleure cardiologie, la plus en pointe ; à côté le dermatologue qui voulait aller le plus loin possible etc.. À l'absence de contrainte économique correspondait l'absence de contrainte aux stratégies individuelles.

14 Les années 80 substituent à ces démarches individualistes un véritable jeu à trois :

15 - «le pôle tutelle " qui va au-delà de l'hôpital et qui impose une politique de santé intégrant les besoins de la population et les contraintes de financement du système de santé

16 - « le pôle hôpital » qui dans un tel contexte doit définir son positionnement par rapport à la politique de santé et définir une stratégie de développement

17 - «le pôle individuel» qui doit persister mais redéfinir ses marques dans le contexte économique et stratégique de cette fin de siècle.

18 C'est donc à une véritable communication de choix auquelle nous conduit l'évolution du contexte dans lequel évolue désormais l'hôpital; en s'efforçant notamment de passer d'une « politique de l'oukase » à une «politique de la gestion raisonnée » conduisant à une rationnalisation des choix. 

cette expression directe des personnels. En effet, on favorise la communication, on permet à chacun d'exprimer ses besoins, ses difficultés, ses peines, parfois ses joies,... mais comment ne pas désespérer des gens qui ont réfléchi, pensé, comment faire en sorte que cela se traduise dans le concret, comment une démarche de communication s'inscrit dans la réalité concrète de l'hôpital?

\section{Qui est cadre à l'hôpital ?}

Cette question est lancinante. C'est une question difficile qui se pose probablement de façon très particulière au sein de l'hôpital par rapport à une entreprise traditionnelle où il est probablement plus facile de dire qui est cadre et qui ne l'est pas. 
28 À l'Assistance Publique-Hôpitaux de Paris, nous avons récemment mené une enquête pour essayer de répondre à cette question. difficulté de positionnement va naturellement se dégager plaçant le plus souvent les cadres prescrits en cadres intermédiaires, les cadres réels disposant quant à eux d'un potentiel de reconnaissance incontestable. positionnement des uns et des autres. La formation constitue probablement un outil majeur de reconnaissance. C'est le cas en particulier de la formation destinée aux cadres supérieurs de l'AP-HP et réalisée en partenariat avec le groupe HEC. Avec l'Ecole de Management des Médecins Hospitaliers (EMAMH), l'AP-HP est en train d'amorcer une approche commune des cadres supérieurs de l'hôpital entre le médecin et le directeur. S'ils ont culturellement des métiers différents, dans leur responsabilité de service, il est clair que des éléments communs se retrouvent entre un chef de service de cardiologie et un directeur des services économiques. cohérence entre les responsables hospitaliers mais l'objectif n'est pas d'en faire des gens semblables qui passeraient par un même moule mais des gens qui ont des préoccupations communes. L'enjeu culturel c'est en permanence cette dialectique entre la recherche de l'unité, de la cohérence et la gestion de la diversité des individualités culturelles.

\section{Recréer la notion d'équipe}

Créer une équipe ce n'est pas si simple à l'hôpital et pourtant c'est fondamental. Soigner des malades c'est avant tout un travail d'équipe. Là aussi, nous entrons dans un autre enjeu de la communication: comment par un meilleur travail en équipe déboucher sur une meilleure prise en charge du malade?

36 Pour bien comprendre la difficulté de cette communication avec le malade, je me référerai à une étude réalisée en 1984 à l'AP-HP, par Kristina ORFAL1, qui débouchera plus tard sur un travail centré sur la relation soignant-soigné. L'intérêt de cette étude est d'expliquer les trois dimensions psycho-sociologiques du patient :

37 - le patient est un malade dans son rapport à la maladie: il est à ce moment-là en complète situation d'assisté, il se livre entièrement à l'équipe médicale à laquelle il ne peut que faire confiance. Dans ce contexte, le patient ne réclame, à la limite, aucune communication sauf une communication de base, d'explication simple. 

branle tout le système de soin. Le patient à ce moment-là ne sera plus seulement un malade mais une personne qui, loin d'être un assisté, deviendra un acteur et revendiquera une certaine communication. Pour prendre un exemple simple : si on va lui fait une piqûre, il voudra savoir pourquoi on la lui fait, il posera des questions à l'infirmière. On est loin d'une communication de base pour rentrer dans une communication forte et complète.

39 un client usager d'un service public, le service public de santé. Par rapport à l'organisation hospitalière, il n'est plus en situation d'assisté mais en situation d'exigence. En effet, le patient comme dans toute administration doit affronter là aussi, par exemple, les tracasseries administratives ou plus spécifiquement à l'hôpital les problèmes de nourriture comme on peut, par exemple les rencontrer à l'hôtel. Ce point est essentiel quand on sait que la plupart des lettres de réclamations que reçoivent les hôpitaux concernent les problèmes d'organisation.

40 Par ailleurs, les difficultés de fonctionnement d'un service hospitalier, au delà des caractéristiques psycho-sociologiques du malade, concernent les questions de relation au sein de l'équipe.

41 Dans un ouvrage («Du mandarin au manager hospitalier ») Marianne BINTZ soulignait que finalement à l'hôpital tout pouvait être organisé au travers de la technique, sans intervention d'une quelconque relation humaine. La technique peut pourvoir à tout : de la prescription à l'administration des médicaments. Or c'est bien là que commencent tous les problèmes de reconnaissance et de communication qui nécessitent de gérer la diversité des métiers en s'efforçant d'intégrer la dimension humaine dans l'organisation du service.

Cette communication de proximité rend nécessaire une réflexion approfondie sur le fonctionnement de l'équipe de soins qui nécessite bien souvent un travail spécifique et différent adapté à la spécificité de chaque service. C'est probablement dans ce domaine, que la communication rencontre la formation. Celle-ci doit remplir trois missions pour améliorer la communication au sein de l'équipe de soin :

- permettre à l'équipe de surmonter la charge psychologique lourde due au contact permanent avec la maladie dans les services hospitaliers. En effet pour ne citer que cet exemple, on a constaté que si les réunions de staff étaient bien organisées, moins de $20 \%$ des services avaient des réunions de service, c'est-à-dire des réunions qui impliquent la présence de tous les métiers (médecins, infirmières, surveillants, etc..) où l'on va aborder des questions qui touchent à la vie du service et qui en elles- mêmes peuvent constituer des facteurs de communication ou des freins à la communication.

Si l'hôpital organise bien la médecine, l'hôpital doit aussi bien organiser ses services, son service au malade. 


\section{Gérer la diversité, gérer les individualités} d'information. Nous avons mené au sein de l'AP-HP une réflexion sur la nature de l'information nécessaire aux uns et aux autres. Cette réflexion nous a mené à nous interroger sur le rôle du journal interne. Doit-il s'adresser à tout le monde, comment peut-il rendre compte de la diversité des préoccupations, comment peut-il coller au mieux aux attentes de tous? objectif de reconnaissance, un objectif d'identité, d'appartenance, un objectif de responsabilité ou de reconnaissance de la responsabilité.

(cest objectifs (c'est celui que nous avons fait à l'AP-HP) réside dans le fait qu'il est difficile par un seul vecteur d'information de toucher tous les publics. Si le journal interne, tel que nous le connaissons dans la plupart des hôpitaux, joue bien son rôle de développement du sentiment d'appartenance, il est évident qu'il ne concerne pas la spécificité des métiers hospitaliers qui dans leur exercice ont probablement besoin chacun d'informations particulières spécifiques à leur domaine. C'est ainsi qu'à l'AP-HP un journal «Flash médical » s'adresse spécifiquement aux médecins. Nous sommes par ailleurs en train de réfléchir à un journal destiné spécifiquement aux cadres, avec toute la difficulté que représente, on l'a dit, la définition de ce terme au sein des hôpitaux.

51 Avec le regard de la communication, il convient de reconnaitre que les cultures au sein de l'hôpital resteront des cultures fortes. Il est donc illusoire de créer un nivellement qui ferait que l'on s'adresse à chacune avec des mots identiques. La richesse de la communication hospitalière est la diversité: diversité des langages permettant la complémentarité des professions.

On le voit bien la notion de journal interne n'est pas morte, mais le journal interne n'est pas un outil unique mais un outil multiple qui repose sur la diversité des supports et des langages, pour mieux faire évoluer les comportements.

En conclusion, je voudrais dire que lorsqu'on fait de la communication, lorsqu'on fait de l'information on doit toujours s'interroger sur la signification de ces mots. Jean-Marie COLOMBANI, directeur du Monde, disait que les hommes de communication transforment la réalité. Je crois qu'un des défis de l'hôpital c'est bien celui-là : sous couvert de communication l'on doit s'efforcer, si l'on veut changer quelque chose, de ne pas enrober la réalité, qui va fatalement resurgir malgré elle.

54 Je plaide donc à l'hôpital pour une communication profonde, une communication de changement culturel, de changement de comportement, une communication qui hélas ne peut avoir de résultats que dans la durée.

\section{Questions}

Mme CARAYOL : Nous allons donner la parole à la salle pour des questions à nos deux intervenants de la matinée.

François BADENES, Chargé de communication à l'hôpital LAENNEC : C 'est une question qui s'adresse à tous les deux, vous avez M. GUYOT développé la dichotomie qu'il y a 
traditionnellement entre le corps médical et le corps administratif. Je crois qu'il s'agit aujourd'hui d'une trichotomie parce qu'il n'y a plus seulement le personnel médical et les administrateurs, il y a aussi tous les cadres intermédiaires, les personnels soignants, le rôle éminemment important des surveillantes générales. Ma question concerne ce rôle des cadres intermédiaires et cette difficulté d'avoir trois corps constitués qui ont du mal à communiquer entre eux, on le voit avec la mise en place difficile des réunions de service qui sont censées elles-mêmes décliner un projet d'établissement qui parait souvent lointain au personnel. Comment rétablir la proximité et comment moduler notre communication vers les surveillantes générales ou vers les cadres dits intermédiaires? Comment moduler cette communication par rapport à ces différents acteurs?

57 Jean-François TOME : Je suis assez sensible à cette notion de trichotomie. C'est vrai il y a un peu de cela. Cependant je répondrai à cette question en deux temps. D'abord, je pense qu'au sein de l'hôpital malgré l'existence fatale de deux logiques de pouvoir, celle du corps médical et celle du corps administratif, on constate aujourd'hui un rapprochement entre ces deux logiques, probablement lié à la prise en compte de nécessités, d'exigences. On voit en effet aujourd'hui de plus en plus de médecins participer à la gestion même si ce phénomène touche davantage les responsables de service que les autres médecins.

Il est important de ne pas négliger cette évolution qui modifie profondément les rapports au sein de l'hôpital. On assiste réellement à une volonté d'aborder les questions hospitalières au travers d'approches communes où les médecins et les responsables administratifs se retrouvent le plus souvent.

À côté du rapprochement médecins / responsables hospitaliers, on doit cependant admettre que l'une des difficultés majeures, réside dans la difficulté de management liée à l'existence à l'hôpital d'une multiplicité de responsables ce qui me fait parfois penser que l'hôpital ressemble à une véritable armée mexicaine où tout le monde est chef.

C'est un phénomène qui touche probablement davantage les services administratifs que les services de soins qui rencontrent quant à eux un problème différent: le cadre infirmier est situé entre deux chaises dans la mesure où il est à la fois gestionnaire et soignant. Comment régler cette question? Je pense qu'elle passe par deux moyens : d'abord la formation qui sans aucun doute va permettre l'évolution des comportements, ensuite une meilleure définition des postes de travail et des missions des uns et des autres.

\section{AUTEUR}

\section{JEAN-FRANÇOIS TOME}

Directeur d'Hôpital, Chef de Service au Département Communication, Assistance Publique-Hôpitaux de Paris 